\title{
Critères de non-automaticité et leurs applications
}

\author{
par \\ JiA-YAN YAO (Talence)
}

I. Introduction. La motivation de cet article est le critère de B. de Mathan (voir le théorème 9). A l'origine, ce critère avait été démontré dans [5] par une méthode d'approximation diophantienne. C'est M. Koskas qui était le premier à donner une démonstration en termes de suites automatiques d'un cas particulier du critère de B. de Mathan (cf. [6]). En examinant sa démonstration, nous avons pris conscience que son idée est fondée sur le théorème 6 . En modifiant légèrement la démonstration due à $B$. de Mathan du théorème 9 , nous obtenons alors le théorème 10. En appliquant le théorème 5 , nous obtenons ensuite une nouvelle démonstration du théorème de M. Mkaouar.

La découverte du lien entre la théorie des approximations diophantiennes et la théorie des suites automatiques conduit à un nouvel essor de ces deux théories. De nombreux résultats sont déjà établis ou sont en train d'être établis. Signalons en particulier que B. de Mathan a donné récemment une version quantitative du théorème 1 .

Rappelons une définition équivalente de la suite automatique. Soient $p \geq 1$ un entier et $u$ une suite à valeurs dans un ensemble fini $S$. La suite $u=(u(n))_{n \geq 0}$ est dite $p$-automatique si le $p$-noyau $\mathcal{N}_{p}(u)$ défini par

$$
\mathcal{N}_{p}(u)=\left\{\left(u\left(p^{a} n+b\right)\right)_{n \geq 0}: a \geq 0,0 \leq b<p^{a}\right\}
$$

est un ensemble fini. Nous remarquons que si $u$ est une suite $p$-automatique, alors elle est aussi $q$-automatique pour tout entier $q \geq 2$ tel que $\log _{p} q \in \mathbb{Q}$.

Il existe un lien étroit entre les suites automatiques et les séries formelles algébriques que nous allons préciser maintenant.

Soient $p \geq 2$ un entier premier et $k \geq 1$ un entier. Posons $q=p^{k}$. Notons $\mathbb{F}_{q}$ le corps à $q$ éléments, $\mathbb{F}_{q}[T]$ l'anneau des polynômes à coefficients dans $\mathbb{F}_{q}$, $\mathbb{F}_{q}(T)$ le corps des fractions rationnelles à coefficients dans $\mathbb{F}_{q}$ et $\mathbb{F}_{q}\left(\left(T^{-1}\right)\right)$ le corps des séries formelles de Laurent à coefficients dans $\mathbb{F}_{q}$. Pour tout $\alpha \in \mathbb{F}_{q}\left(\left(T^{-1}\right)\right)$, nous écrivons 


$$
\alpha=\sum_{j=j_{0}}^{\infty} \alpha(j) T^{-j} \quad \text { avec } \alpha\left(j_{0}\right) \neq 0 .
$$

Posons $v_{\infty}(\alpha)=-j_{0}$ et définissons $|\alpha|=p^{v_{\infty}(\alpha)}$. Soit maintenant $\Delta$ un polynôme irréductible à coefficients dans $\mathbb{F}_{q}$. Pour tout polynôme $P$ dans $\mathbb{F}_{q}[T]$, désignons $v_{\Delta}(P)$ le plus grand entier $k$ tel que $\Delta^{k}$ divise $P$ dans l'anneau $\mathbb{F}_{q}[T]$. Nous disons qu'une série formelle $\alpha \in \mathbb{F}_{q}\left(\left(T^{-1}\right)\right)$ est algébrique si elle est algébrique sur le corps $\mathbb{F}_{q}(T)$. Dans le cas contraire, nous disons que $\alpha$ est transcendante.

Dans [3], G. Christol, T. Kamae, M. Mendès France et G. Rauzy ont démontré le résultat suivant, qui est la base de toutes nos démonstrations.

ThÉORÈme 1 . Soit u une suite à valeurs dans $\mathbb{F}_{q}$. Alors u est $p$-automatique si et seulement si la série formelle $\sum_{n=0}^{\infty} u(n) T^{-n}$ est algébrique sur $\mathbb{F}_{q}(T)$.

II. Critères de non-automaticité. Soit $u$ une suite à termes dans un ensemble fini $S$. Pour tout $a \in S$, posons

$$
\bar{d}(u, a)=\limsup _{N \rightarrow \infty} \frac{1}{N} \operatorname{Card}(\{0 \leq n<N: u(n)=a\}) .
$$

Ainsi $\bar{d}(u, a)$ est la densité supérieure des occurrences de $a$ dans la suite $u$.

Nous citons sans démonstration le théorème suivant d'A. Cobham (cf. [4]) :

ThÉORÈme 2. Soient $p \geq 2$ un entier et $u$ une suite p-automatique $\grave{a}$ termes dans un ensemble fini $S$. Fixons $a \in S$. Alors $\bar{d}(u, a)=0$ si, et seulement si, il existe deux entiers $s \geq 1$ et $t \geq 1$ tels que $p^{s-1} \leq t<p^{s}$ et $u(n) \neq a$ pour tout entier $n$ de la forme $n=j p^{s+k}+t p^{k}+l$ avec $j, k \geq 0$ et $0 \leq l<p^{k}$.

Le résultat ci-dessous est dû essentiellement à M. Mkaouar (cf. [9]) :

ThÉORÈme 3. Soient u une suite à termes dans un ensemble fini $S$ et $a \in S$ tels que $\bar{d}(u, a)=0$. Soit $(b(n))_{n \geq 0}$ une suite strictement croissante d'entiers telle que pour tout entier $n \geq 0$, nous avons $u(b(n))=a$. Supposons qu'il existe un entier $p \geq 2$ et un nombre $c>0$ tels que

$$
b(n) \sim c p^{n} \quad \text { quand } n \rightarrow \infty .
$$

Alors pour tout entier $q \geq 2$ tel que $\log _{p} q \notin \mathbb{Q}$, la suite $u$ n'est pas $q$ automatique.

Démonstration. Fixons un entier $q \geq 2$ tel que $\log _{p} q \notin \mathbb{Q}$. Quitte à remplacer $q$ par une puissance de $q$, nous pouvons supposer $q>p$ sans perte de généralité. Pour tout $n \geq 0$, posons $b(n)=c(n) p^{n}$. Quitte à remplacer $c$ par $c p^{N}$ et $b(n)$ par $b(n+N)$, nous supposons $c>1$. 
Soient $t \geq 1$ et $s \geq 1$ deux entiers tels que $q^{s-1} \leq t<q^{s}$. Montrons qu'il existe un entier $n$ tel que $b(n)=q^{s+k}+t q^{k}+l$ avec $k \geq 0$ et $0 \leq l<q^{k}$. Cela suffit à conclure que la suite $u$ n'est pas $q$-automatique (voir le théorème 2 ).

Soit $\delta$ un nombre réel tel que $0<\delta<\left(2 q^{s}+2 t+1 / c\right)^{-1}$. Par suite,

$$
\frac{q^{s}+t}{c-\delta}<\frac{q^{s}+t+1 / c}{c+\delta} .
$$

Comme $\lim _{n \rightarrow \infty} c(n)=c>1$, il existe alors un entier $n_{0} \geq 0$ tel que

$$
1 / c<1-q^{-n} \quad \text { et } \quad c-\delta \leq c(n) \leq c+\delta \quad \text { pour tout entier } n \geq n_{0} .
$$

D'après l'hypothèse du théorème, nous avons $\log _{p} q \notin \mathbb{Q}$. La suite $\left(n \log _{p} q\right)_{n>n_{0}}$ est donc équirépartie modulo 1 (cf. [7]). Il existe ainsi deux entiers $n, k \geq n_{0}$ tels que

$$
\log _{p}\left(\frac{q^{s}+t}{c-\delta}\right)<n-k \log _{p} q<\log _{p}\left(\frac{q^{s}+t+1 / c}{c+\delta}\right) .
$$

Nous en déduisons immédiatement l'inégalité suivante :

$$
\log _{p}\left(\frac{q^{s}+t}{c(n)}\right)<n-k \log _{p} q<\log _{p}\left(\frac{q^{s}+t+1-q^{-k}}{c(n)}\right) .
$$

Par suite $\left(q^{s}+t\right) q^{k}<b(n)=c(n) p^{n}<\left(q^{s}+t+1\right) q^{k}-1$.

Soient $p \geq 2$ un entier et $u$ une suite arbitraire. Pour $a \geq 0$ et $0 \leq b<p^{a}$, posons

$$
{ }_{p} u^{a, b}(n)=u\left(p^{a} n+b\right), \quad \forall n \in \mathbb{N} .
$$

ThÉORÈme 4. Soient $u$ une suite à valeurs dans un ensemble fini $S$ et $a \in S$. Soient $(l(n))_{n \geq 0}$ et $(h(n))_{n \geq 0}$ deux suites strictement croissantes d'entiers vérifiant:

(1) pour tout $n \in \mathbb{N}, h(n) \leq l(n)$ et $u(h(n)-1) \neq a$;

(2) pour tout $n \in \mathbb{N}, u(m)=a$ pour tout $h(n) \leq m \leq l(n)$;

(3) $\lim _{n \rightarrow \infty} l(n) / h(n)=+\infty$.

Alors u n'est pas une suite automatique.

Démonstration. Soit $p \geq 2$ un entier. Pour tout entier $n \geq 0$, posons

$$
k(n)=\left\lfloor\log _{p} \frac{l(n)}{h(n)}\right\rfloor .
$$

Considérons le développement en base $p$ de l'entier $h(n)-1$ :

$$
h(n)-1=\sum_{j=0}^{a(n)} h_{j}(n) p^{j} .
$$


Pour tout entier $0 \leq k \leq d(n):=\min (k(n)-1, a(n))$, définissons $b_{k}(n)$, $c_{k}(n)$ par

$$
b_{k}(n)=\sum_{j=0}^{a(n)-k} h_{j+k}(n) p^{j} \quad \text { et } \quad c_{k}(n)=\sum_{j=0}^{k-1} h_{j}(n) p^{j}
$$

de sorte que $h(n)-1=b_{k}(n) p^{k}+c_{k}(n)$. Nous obtenons par suite,

${ }_{p} u^{k, c_{k}(n)}\left(b_{k}(n)\right) \neq a \quad$ et $\quad{ }_{p} u^{k, c_{k}(n)}(m)=a \quad$ avec $h(n) \leq p^{k} m+c_{k}(n) \leq l(n)$.

Soient $0 \leq s<t \leq d(n)$ deux entiers. Alors d'une part ${ }_{p} u^{s, c_{s}(n)}\left(b_{s}(n)\right) \neq a$, et d'autre part ${ }_{p} u^{t, c_{t}(n)}\left(b_{s}(n)\right)=a$ car $h(n) \leq p^{t} b_{s}(n)+c_{t}(n) \leq l(n)$. Par conséquent,

$$
\operatorname{Card}\left(\mathcal{N}_{p}(u)\right) \geq d(n) \quad \text { pour tout } n \geq 0 \text {. }
$$

Comme $(d(n))_{n \geq 0}$ n'est pas bornée, $u$ n'est pas $p$-automatique.

\section{II.1. Critères de non-automaticité de type I}

ThÉORÈme 5. Soient u une suite à termes dans un ensemble fini $S$ et a un élément de $S$. Soient $(l(n))_{n \geq 0}$ et $(h(n))_{n \geq 0}$ deux suites strictement croissantes d'entiers telles que pour tout entier $n \geq 0, h(n) \leq l(n)$ et

$$
u(m)=a \quad \text { pour tout } h(n) \leq m \leq l(n) \quad \text { et } \quad u(h(n)-1) \neq a .
$$

Soit $p \geq 2$ un entier. Supposons que pour tout entier $N \geq 1$, nous avons

$$
\lim _{n \rightarrow \infty}\left(l(n+N)-p^{N} h(n)\right)=+\infty \quad \text { et } \quad \lim _{n \rightarrow \infty}\left(p^{N} h(n)-h(n+N)\right)=+\infty .
$$

Alors u n'est pas une suite p-automatique.

Démonstration. Soit $n \geq 0$ un entier. D'après la définition, nous avons

$$
u(h(n)-1) \neq a \quad \text { et } \quad h(k)=a \quad \text { pour } h(n) \leq k \leq l(n) .
$$

Considérons le développement en base $p$ de l'entier $h(n)-1$ :

$$
h(n)-1=\sum_{j=0}^{a(n)} h_{j}(n) p^{j} .
$$

Pour tout entier $0 \leq k \leq a(n)$, définissons deux entiers $b_{k}(n), c_{k}(n)$ par

$$
b_{k}(n)=\sum_{j=0}^{a(n)-k} h_{j+k}(n) p^{j} \quad \text { et } \quad c_{k}(n)=\sum_{j=0}^{k-1} h_{j}(n) p^{j} .
$$

Ainsi $h(n)-1=b_{k}(n) p^{k}+c_{k}(n)$ et nous avons

${ }_{p} u^{k, c_{k}(n)}\left(b_{k}(n)\right) \neq a \quad$ et $\quad{ }_{p} u^{k, c_{k}(n)}(m)=a \quad$ avec $h(n) \leq p^{k} m+c_{k}(n) \leq l(n)$. 
Soit $N \geq 1$ un entier. Il existe alors $n \geq 0$ tel que pour tout entier $1 \leq k \leq N$,

$$
p^{k} h(n)+p^{N} \leq l(n+k) \quad \text { et } h(n+k)+p^{N} \leq p^{k}(h(n)-1) .
$$

Fixons deux entiers $1 \leq s<t \leq N$. Les inégalités précédentes donnent

$h(n+t-s) \leq p^{t} b_{s}(n)+c_{t}(n)=p^{t-s}(h(n)-1)+c_{t}(n)-p^{t-s} c_{s}(n) \leq l(n+t-s)$.

Nous en déduisons immédiatement les relations suivantes :

$$
{ }_{p} u^{s, c_{s}(n)}\left(b_{s}(n)\right) \neq a \quad \text { et } \quad{ }_{p} u^{t, c_{t}(n)}\left(b_{s}(n)\right)=a \quad \text { pour tout } 1 \leq s<t \leq N .
$$

Ainsi $\operatorname{Card}\left(\mathcal{N}_{p}(u)\right) \geq N$. Par conséquent, la suite $u$ n'est pas $p$-automatique.

THÉORÈME 6. Soient $u$ une suite à termes dans un ensemble fini $S$ et a un élément de $S$. Soient $(l(n))_{n \geq 0}$ et $(h(n))_{n \geq 0}$ deux suites strictement croissantes d'entiers telles que pour tout entier $n \geq 0, h(n) \leq l(n)$ et

$$
u(m)=a \quad \text { pour tout } h(n) \leq m \leq l(n) \quad \text { et } \quad u(h(n)-1) \neq a .
$$

Soit $p \geq 2$ un entier. Supposons que pour tout entier $N \geq 1$, nous avons

$$
\lim _{n \rightarrow \infty}\left(p^{N} l(n-N)-h(n)\right)=+\infty \quad \text { et } \quad \lim _{n \rightarrow \infty}\left(h(n)-p^{N} h(n-N)\right)=+\infty .
$$

Alors u n'est pas une suite p-automatique.

Démonstration. Nous gardons les notations du théorème précédent. Soit $N \geq 1$ un entier. Il existe alors $n \geq 0$ tel que pour tout entier $1 \leq k \leq N$,

$$
p^{-k} h(n)+p^{N} \leq l(n-k) \quad \text { et } h(n-k)+p^{N} \leq p^{-k}(h(n)-1) .
$$

Fixons deux entiers $1 \leq s<t \leq N$. Les inégalités précédentes donnent $h(n+s-t) \leq p^{s} b_{t}(n)+c_{s}(n)=p^{s-t}(h(n)-1)+c_{s}(n)-p^{s-t} c_{t}(n) \leq l(n+s-t)$.

Nous en déduisons immédiatement les relations suivantes :

$$
{ }_{p} u^{t, c_{t}(n)}\left(b_{t}(n)\right) \neq a \quad \text { et } \quad{ }_{p} u^{s, c_{s}(n)}\left(b_{t}(n)\right)=a \text { pour tout } 1 \leq s<t \leq N .
$$

Ainsi $\operatorname{Card}\left(\mathcal{N}_{p}(u)\right) \geq N$. Par conséquent, la suite $u$ n'est pas $p$-automatique.

II.2. Critères de non-automaticité de type II. Dans les résultats du paragraphe précédent, nous utilisons constamment le fait que

$$
u(h(n)-1) \neq a \quad \text { pour tout } n \geq 0 .
$$

Cela nous oblige à imposer une relation entre $h(n)$ et $h(n+1)$. Par contre, nous n'avons besoin d'aucune relation entre $l(n)$ et $l(n+1)$. Dans ce paragraphe, nous donnons un autre type de critère de la non-automaticité qui consiste à intervertir les rôles joués par les deux suites d'entiers $(h(n))_{n \geq 0}$ et $(l(n))_{n \geq 0}$. 
ThÉORÈme 7. Soient u une suite à termes dans un ensemble fini $S$ et a un élément de $S$. Soient $(l(n))_{n \geq 0}$ et $(h(n))_{n \geq 0}$ deux suites strictement croissantes d'entiers telles que pour tout entier $n \geq 0, h(n) \leq l(n)$ et

$$
u(m)=a \quad \text { pour tout } h(n) \leq m \leq l(n) \quad \text { et } \quad u(l(n)+1) \neq a .
$$

Soit $p \geq 2$ un entier. Si pour tout entier $N \geq 1$, nous avons

$$
\lim _{n \rightarrow \infty}\left(l(n+N)-p^{N} h(n)\right)=+\infty \text { et } \lim _{n \rightarrow \infty}\left(p^{N} l(n)-l(n+N)\right)=+\infty,
$$

alors $u$ n'est pas une suite p-automatique.

Démonstration. Soit $n \geq 0$ un entier. D'après la définition, nous avons

$$
u(l(n)+1) \neq a \quad \text { et } \quad h(k)=a \quad \text { pour } h(n) \leq k \leq l(n) .
$$

Considérons le développement en base $p$ de l'entier $l(n)+1$ :

$$
l(n)+1=\sum_{j=0}^{a(n)} l_{j}(n) p^{j} .
$$

Pour tout entier $0 \leq k \leq a(n)$, définissons deux entiers $b_{k}(n), c_{k}(n)$ par

$$
b_{k}(n)=\sum_{j=0}^{a(n)-k} l_{j+k}(n) p^{j} \quad \text { et } \quad c_{k}(n)=\sum_{j=0}^{k-1} l_{j}(n) p^{j} .
$$

Alors $l(n)+1=b_{k}(n) p^{k}+c_{k}(n)$ et nous avons

${ }_{p} u^{k, c_{k}(n)}\left(b_{k}(n)\right) \neq a \quad$ et $\quad{ }_{p} u^{k, c_{k}(n)}(m)=a \quad$ avec $h(n) \leq p^{k} m+c_{k}(n) \leq l(n)$.

Soit $N \geq 1$ un entier. Il existe alors $n \geq 0$ tel que pour tout entier $1 \leq k \leq N$,

$$
h(n-k)+p^{N} \leq p^{-k}(l(n)-1) \quad \text { et } \quad p^{-k} l(n)+p^{N} \leq l(n-k) .
$$

Fixons deux entiers $1 \leq t<s \leq N$. Les inégalités précédentes nous donnent

$$
\begin{aligned}
h(n+t-s) & \leq p^{t} b_{s}(n)+c_{t}(n)=p^{t-s}(l(n)-1)+c_{t}(n)-p^{t-s} c_{s}(n) \\
& \leq l(n+t-s) .
\end{aligned}
$$

Nous en déduisons immédiatement les relations suivantes :

$$
{ }_{p} u^{s, c_{s}(n)}\left(b_{s}(n)\right) \neq a \quad \text { et } \quad{ }_{p} u^{t, c_{t}(n)}\left(b_{s}(n)\right)=a \quad \text { pour tout } 1 \leq t<s \leq N .
$$

Ainsi $\operatorname{Card}\left(\mathcal{N}_{p}(u)\right) \geq N$. Par conséquent, la suite $u$ n'est pas $p$-automatique.

THÉORÈME 8. Soit u une suite à termes dans un ensemble fini $S$ et $a$ un élément de $S$. Soient $(l(n))_{n \geq 0}$ et $(h(n))_{n \geq 0}$ deux suites strictement croissantes d'entiers telles que pour tout entier $n \geq 0, h(n) \leq l(n)$ et

$$
u(m)=a \quad \text { pour tout } h(n) \leq m \leq l(n) \quad \text { et } \quad u(l(n)+1) \neq a .
$$


Soit $p \geq 2$ un entier. Si pour tout entier $N \geq 1$, nous avons

$$
\lim _{n \rightarrow \infty}\left(p^{N} l(n)-h(n+N)\right)=+\infty \text { et } \lim _{n \rightarrow \infty}\left(l(n+N)-p^{N} l(n)\right)=+\infty,
$$

alors $u$ n'est pas une suite p-automatique.

Démonstration. Gardons les notations du théorème précédent. Soit $N \geq 1$ un entier. Il existe alors un entier $n \geq 0$ tel que pour tout entier $1 \leq k \leq N$,

$$
h(n+k)+p^{N} \leq p^{k}(l(n)-1) \quad \text { et } \quad p^{k} l(n)+p^{N} \leq l(n+k) .
$$

Fixons deux entiers $1 \leq t<s \leq N$. Les inégalités précédentes nous donnent

$$
\begin{aligned}
h(n+s-t) & \leq p^{s} b_{t}(n)+c_{s}(n)=p^{s-t}(l(n)-1)+c_{s}(n)-p^{s-t} c_{t}(n) \\
& \leq l(n+s-t) .
\end{aligned}
$$

Nous en déduisons immédiatement les relations suivantes :

$$
{ }_{p} u^{t, c_{t}(n)}\left(b_{t}(n)\right) \neq a \quad \text { et } \quad{ }_{p} u^{s, c_{s}(n)}\left(b_{t}(n)\right)=a \quad \text { pour tout } 1 \leq t<s \leq N .
$$

Ainsi $\operatorname{Card}\left(\mathcal{N}_{p}(u)\right) \geq N$. Par conséquent, $u$ n'est pas une suite $p$-automatique.

III. Applications. Les théorèmes exposés dans les deux paragraphes précédents possèdent de nombreuses applications ainsi que l'a remarqué B. de Mathan (voir [5]). Nous en donnons deux autres dans la suite.

III.1. Critère de transcendance de B. de Mathan. Nous citons sans démonstration le critère de B. de Mathan suivant (cf. [5] ou [6]) :

ThÉORÈme 9. Soit $\alpha \in \mathbb{F}_{q}\left(\left(T^{-1}\right)\right)$. Supposons qu'il existe une suite $\left(P_{n} / Q_{n}\right)_{n>0}$ d'approximations rationnelles de $\alpha$ où $P_{n}$ et $Q_{n}$ sont éléments de $\mathbb{F}_{q}[T]$, avec $P_{n} Q_{n} \neq 0\left(P_{n}\right.$ et $Q_{n}$ ne sont pas nécessairement premiers entre eux), vérifiant les conditions :

(1) il existe $\Lambda \in \mathbb{F}_{q}[T]$ tel que $Q_{n}=\Lambda Q_{n-1}^{q}$ pour tout $n \geq 1$,

(2) $\left|\alpha-P_{n} / Q_{n}\right|<C\left|Q_{n}\right|^{-1}$ avec $C>0$ une constante,

(3) il existe $\Delta \in \mathbb{F}_{q}[T]$ irréductible tel que

$$
\lim _{n \rightarrow \infty}\left(q v_{\Delta}\left(P_{n-1}\right)-v_{\Delta}\left(P_{n}\right)\right)=+\infty .
$$

Alors la série formelle de Laurent $\alpha$ est transcendante.

Montrons que dans le cas où $Q_{0}=1, \Lambda=T^{s}$ et $\Delta=T$, le critère de B. de Mathan est un corollaire du théorème 6 . Pour tout entier $n \geq 0$, posons $\alpha_{n}=P_{n} / Q_{n}$. Quitte à multiplier $\alpha$ par une puissance de $T$, nous supposons $v_{\infty}(\alpha)=0$. Par conséquent, quand l'entier $n$ devient assez grand, nous avons $p(n):=\operatorname{deg} P_{n}=\operatorname{deg} Q_{n}=s\left(q^{n}-1\right) /(q-1)$ en vertu de la condition (2). 
Notons $u$ (resp. $u_{n}$ ) la suite des coefficients de la série formelle $\alpha$ (resp. $\left.\alpha_{n}\right)$. Désignons par $l(n)$ le plus grand entier $m$ tel que pour $0 \leq k \leq m$, $u(k)=u_{n}(k)$. D'après la condition (2), il existe un entier constant $c \geq 0$ tel que $l(n) \geq p(n)-c$. Posons $h(n)=p(n)-v_{T}\left(P_{n}\right)+1$. Il est clair que si $n$ est assez grand, $h(n)$ est le plus petit entier $m$ tel que $u(l)=0$ pour tout $m \leq l \leq l(n)$. Nous vérifions sans peine que les deux suites $(l(n))_{n \geq 0}$ et $(h(n))_{n \geq 0}$ satisfont aux conditions du théorème 6 . Ainsi $\alpha$ est transcendante en vertu du théorème 1 .

Il est facile de traduire les critères de non-automaticité exposés dans les paragraphes précédents dans le langage des séries formelles. Inspiré par le critère de transcendance de $\mathrm{B}$. de Mathan, nous avons obtenu le résultat suivant :

THÉORÈme 10. Soit $\alpha \in \mathbb{F}_{q}\left(\left(T^{-1}\right)\right)$. Supposons qu'il existe une suite $\left(P_{n} / Q_{n}\right)_{n \geq 0}$ d'approximations rationnelles de $\alpha$ où $P_{n}$ et $Q_{n}$ sont éléments de $\mathbb{F}_{q}[T]$, avec $P_{n} Q_{n} \neq 0\left(P_{n}\right.$ et $Q_{n}$ ne sont pas nécessairement premiers entre eux), vérifiant les conditions:

(1) il existe $\Lambda \in \mathbb{F}_{q}[T]$ tel que $Q_{n}=\Lambda Q_{n-1}^{q}$ pour tout $n \geq 1$,

(2) $\left|\alpha-P_{n} / Q_{n}\right|<C\left|Q_{n}\right|^{-1}$ avec $C>0$ une constante,

(3) il existe $\Delta \in \mathbb{F}_{q}[T]$ irréductible tel que

$$
\lim _{n \rightarrow \infty}\left(v_{\Delta}\left(P_{n}\right)-q v_{\Delta}\left(P_{n-1}\right)\right)=+\infty .
$$

Alors la série formelle de Laurent $\alpha$ est transcendante.

Démonstration. Raisonnons par l'absurde. Supposons que $\alpha$ soit algébrique. Il existe alors un entier $r \geq 1$ et $A_{0}, A_{1}, \ldots, A_{r}$ non tous nuls dans $\mathbb{F}_{q}[T]$ tels que

$$
\beta:=A_{0} \alpha+A_{1} \alpha^{q}+\ldots+A_{r} \alpha^{q^{r}}=0 .
$$

Pour tout entier $n \geq r$, posons

$$
\beta_{n}=A_{0}\left(\frac{P_{n}}{Q_{n}}\right)+A_{1}\left(\frac{P_{n-1}}{Q_{n-1}}\right)^{q}+\ldots+A_{r}\left(\frac{P_{n-r}}{Q_{n-r}}\right)^{q^{r}} .
$$

Nous avons alors $\beta_{n}=B_{n} / Q_{n}$ avec $B_{n}=A_{0} P_{n}+\ldots+A_{r} \Lambda^{\left(q^{r}-1\right) /(q-1)} P_{n-r}^{q^{r}}$. D'après l'hypothèse du théorème, nous avons alors

$$
\lim _{n \rightarrow \infty}\left(v_{\Delta}\left(P_{n}\right)-q v_{\Delta}\left(P_{n-1}\right)\right)=+\infty .
$$

Nous en déduisons immédiatement

$$
v_{\Delta}\left(B_{n}\right)=\max _{0 \leq j \leq r} v_{\Delta}\left(A_{j} \Lambda^{\left(q^{j}-1\right) /(q-1)} P_{n-j}^{q^{j}}\right) \rightarrow+\infty \quad \text { quand } n \rightarrow \infty .
$$

En particulier, la suite $\left(\operatorname{deg}\left(B_{n}\right)\right)_{n \geq r}$ n'est pas bornée. 
Posons $a=\max _{0 \leq j \leq r} \operatorname{deg}\left(A_{j}\right)$. Tenant compte de la condition (2), nous pouvons trouver une constante $c>0$ assez grande telle que

$$
\left|\beta-\beta_{n}\right| \leq|T|^{a} \max _{0 \leq j \leq r}\left|\alpha^{q^{j}}-\left(P_{n-j} / Q_{n-j}\right)^{q^{j}}\right| \leq c\left|Q_{n}\right|^{-1} .
$$

Ainsi $\left|B_{n}\right| \leq c$. La suite $\left(\operatorname{deg}\left(B_{n}\right)\right)_{n \geq r}$ est donc bornée. C'est absurde.

III.2. Série formelle de Baum-Sweet et fraction continue. Comme dans le cas réel, les séries formelles de Laurent peuvent aussi se développer en fraction continue dont les quotients partiels sont des polynômes à coefficients dans le corps fini $\mathbb{F}_{q}$ (cf. [1] et ses références).

Nous rappelons que nous ne connaissons aucun nombre réel algébrique de degré $\geq 3$ dont le développement en fraction continue est à quotients partiels bornés. Plus de choses sont connues dans le cas des séries formelles algébriques : en 1976, L. Baum et M. Sweet ont donné dans [2] le premier exemple d'un élément cubique dont les quotients partiels ne prennent qu'un nombre fini de valeurs ainsi que des exemples dont les quotients partiels prennent une infinité de valeurs. Conscient du théorème 1, M. Mendès France a posé la question naturelle suivante : si les quotients partiels d'une série formelle algébrique ne prennent qu'un nombre fini de valeurs, forment-ils eux aussi une suite automatique? On voit que la réponse à cette question suppose qu'on sache, d'une part déterminer les séries formelles algébriques à quotients partiels dans un ensemble fini, d'autre part, pour chaque série formelle algébrique à quotients partiels dans un ensemble fini, donner une expression (explicite) de ces quotients partiels, enfin montrer l'automaticité de cette suite en utilisant ce développement explicite.

En 1986, W. H. Mills et D. P. Robbins ont donné des exemples de telles séries avec des développements en fractions continues explicites. En effet, ils sont les seuls exemples connus en caractéristique $p>2$ (cf. [8]). En 1988, J.-P. Allouche et al. ont montré que pour chaque exemple donné dans [8] avec $p \geq 3$, la suite des quotients partiels est $p$-automatique. Mais la méthode utilisée ne permettait pas d'étudier l'exemple initial donné en caractéristique 2 par L. Baum et M. Sweet. En utilisant les théorèmes des paragraphes précédents, nous montrons que pour cette série, la suite des quotients partiels n'est pas automatique. Ce résultat avait été démontré par M. Mkaouar (voir [9]), mais sa démonstration est un peu plus compliquée que la nôtre.

Soient $B=\left\{T, T+1, T^{2}, T^{2}+1\right\} \subseteq \mathbb{F}_{2}[T]$ et $w=(w(j))_{0 \leq j \leq m}$ un mot sur $B$. Posons $w^{+}=w(m) \ldots w(1) w(0)$ et $t(w)=(w(0)+1) w(1) \ldots w(m)$. Pour tout $n \geq 1$ et tout mot $w$ sur $B$, désignons $(w)^{n}$ le mot composé de $n$ fois $w$. Avec ces notations, nous allons définir une suite infinie $\Gamma_{\infty}$.

Pour tout entier impair $n \geq 1$, notons $\Lambda_{n}$ la suite palindromique

$$
\Lambda_{n}=T, T+1, T^{2},\left(T, T^{2}\right)^{\left(2^{n-1}-4\right) / 3}, T+1, T .
$$


Pour tout entier pair $n \geq 4$, notons $\Lambda_{n}$ la suite palindromique

$$
\Lambda_{n}=T+1, T^{2}+1,\left(T, T^{2}\right)^{\left(2^{n-1}-5\right) / 3}, T, T^{2}+1, T+1 .
$$

Nous définissons finalement par récurrence la suite de mots $\left(\Gamma_{n}\right)_{n \geq 0}$ par :

$$
\begin{aligned}
& \Gamma_{0}=T+1, \\
& \Gamma_{1}=\Gamma_{0}, T^{2}+1, \\
& \Gamma_{2}=\Gamma_{1}, T, T^{2}, T+1, \\
& \Gamma_{n}=\Gamma_{n-1}, t\left(\Gamma_{n-3}^{+}\right), \Lambda_{n}, \Gamma_{n-3} \text { pour } n \geq 3 .
\end{aligned}
$$

Pour tout entier $n \geq 0$, posons $u(n)=\left|\Gamma_{n}\right|$. Dans [9], M. Mkaouar a montré que la suite d'entiers $(u(n))_{n \geq 0}$ est donnée par la formule suivante :

$$
u(n)=\frac{1}{3} 2^{n+2}+\lambda_{1} r_{1}^{n}+\lambda_{2} r_{2}^{n}+\lambda_{3} r_{3}^{n}+\frac{1}{12}(-1)^{n+1}-1,
$$

où $\lambda_{l}=\frac{1}{116}\left(26+10 r_{1}-r_{l}^{2}\right)(1 \leq l \leq 3)$ et $\left(r_{1}, r_{2}, r_{3}\right)$ sont les trois solutions de l'équation $r^{3}-r^{2}-2=0$.

Plus précisément, nous avons les expressions explicites :

$$
\begin{aligned}
& r_{1}=\frac{\left(28-\sqrt{(28)^{2}-1}\right)^{1 / 3}}{3}+\frac{\left(28+\sqrt{(28)^{2}-1}\right)^{1 / 3}}{3}+\frac{1}{3}, \\
& r_{2}=\frac{\left(28-\sqrt{(28)^{2}-1}\right)^{1 / 3}}{3} \omega+\frac{\left(28+\sqrt{(28)^{2}-1}\right)^{1 / 3}}{3} \omega^{2}+\frac{1}{3}, \\
& r_{3}=\frac{\left(28-\sqrt{(28)^{2}-1}\right)^{1 / 3}}{3} \omega^{2}+\frac{\left(28+\sqrt{(28)^{2}-1}\right)^{1 / 3}}{3} \omega+\frac{1}{3},
\end{aligned}
$$

avec

$$
\omega=-\frac{1}{2}+\frac{\sqrt{3}}{2} i \quad \text { et } \quad \omega^{2}=-\frac{1}{2}-\frac{\sqrt{3}}{2} i .
$$

Nous vérifions sans peine que $1<\left|r_{2}\right|=\left|r_{3}\right|<r_{1}<2$ et $\lambda_{1}>0$.

Comme $\Gamma_{n}$ commence par $\Gamma_{n-1}$, alors la suite de mots $\left(\Gamma_{n}\right)_{n>1}$ admet une limite faible, notée $\Gamma_{\infty}$. Dans [8], W. H. Mills et D. P. Robbins ont montré que le développement en fraction continue de la série formelle de Baum-Sweet $f$ est $f=\left[1, T, \Gamma_{\infty}\right]$. Nous rappelons que la série formelle $f$ est solution de l'équation $T f^{3}+f+T=0$.

ThÉORÈme 11. La suite $\Gamma_{\infty}$ n'est pas automatique.

Dém on s trati on. Soit $\varphi$ l'application à valeurs dans $\{0,1\}$ définie sur $B$ par

$$
\varphi(T)=\varphi\left(T^{2}\right)=0 \quad \text { et } \quad \varphi(T+1)=\varphi\left(T^{2}+1\right)=1 .
$$

Posons $\varphi\left(\Gamma_{\infty}\right)=\left(\varphi\left(\Gamma_{\infty}(n)\right)\right)_{n \geq 0}$. Nous allons montrer que la suite $\varphi\left(\Gamma_{\infty}\right)$ n'est pas automatique. Et cela suffit pour conclure. 
Soit $p \geq 2$ un entier. Il existe alors un entier $k \geq 1$ tel que $2^{k} \leq p<2^{k+1}$. Considérons les deux cas possibles suivants :

$1^{\text {er }}$ cas $: p=2^{k}$. Pour tout entier $n \geq 0$, posons

$$
l(n)=u(k n-1)+u(k n-3)+\left|\Lambda_{k n}\right|-3 .
$$

Alors $\varphi\left(\Gamma_{\infty}\right)(l(n))=0$. Notons $h(n)$ le plus petit entier $m$ tel que

$$
\varphi\left(\Gamma_{\infty}\right)(s)=0 \quad \text { pour tout } m \leq s \leq l(n) .
$$

En étudiant les structures des deux suites finies $\Lambda_{k n}$ et $\Gamma_{k n}$, nous obtenons

$$
\begin{aligned}
& l(n)=\left(\frac{7}{3} 2^{k n-1}+\lambda_{1}\left(r_{1}^{2}+1\right) r_{1}^{k n-3}\right)(1+o(1)) \quad \text { quand } n \rightarrow \infty, \\
& h(n)=\left(\frac{5}{3} 2^{k n-1}+\lambda_{1}\left(r_{1}^{2}+1\right) r_{1}^{k n-3}\right)(1+o(1)) \quad \text { quand } n \rightarrow \infty .
\end{aligned}
$$

Montrons que les deux suites d'entiers $(l(n))_{n \geq 0}$ et $(h(n))_{n \geq 0}$ satisfont aux conditions du théorème 5 . Soit $N \geq 1$ un entier. Nous avons alors

$$
l(n+N)-2^{k N} h(n)=\frac{1}{3} 2^{k(n+N)}(1+o(1)) \rightarrow+\infty \quad \text { quand } n \rightarrow \infty .
$$

D'autre part, en remarquant que $2^{k}>r_{1}^{k}$, nous avons donc

$$
2^{k N} h(n)-h(n+N)=\left(2^{k N}-r_{1}^{k N}\right) \lambda_{1}\left(r_{1}^{2}+1\right) r_{1}^{k n-3}(1+o(1)) \rightarrow+\infty
$$

quand $n \rightarrow \infty$. Ainsi la suite $\varphi\left(\Gamma_{\infty}\right)$ n'est pas $2^{k}$-automatique en vertu du théorème 5 .

$2^{\text {ième }}$ c as : $p$ n'est pas une puissance de 2 . Alors $\log _{2} p \notin \mathbb{N}$ et par suite $\log _{2} p \notin \mathbb{Q}$. D'après la définition de $h(n)$, nous avons ainsi

$$
\varphi\left(\Gamma_{\infty}\right)(h(n)-1)=1 \quad \text { pour tout } n \geq 0 .
$$

Nous vérifions sans peine que $\bar{d}\left(\Gamma_{\infty}, 1\right)=0$. Or

$$
h(n)-1=\frac{5}{3} 2^{k n-1}(1+o(1)) \quad \text { quand } n \rightarrow \infty,
$$

la suite $\varphi\left(\Gamma_{\infty}\right)$ n'est donc pas $p$-automatique en vertu du théorème 3.

Remerciement. Nous remercions chaleureusement Bernard de Mathan et Michel Mendès France pour leurs précieux remarques et conseils.

\section{Références}

[1] J.-P. Allouche, Sur le développement en fraction continue de certaines séries formelles, C. R. Acad. Sci. Paris 307 (1988), 631-633.

[2] L. Baum and M. Sweet, Continued fractions of algebraic power series in characteristic 2, Ann. of Math. 103 (1976), 593-610.

[3] G. Christol, T. Kamae, M. Mendès France et G. Rauzy, Suites algébriques, automates et substitutions, Bull. Soc. Math. France 108 (1980), 401-419.

[4] A. Cobham, Uniform tag sequences, Math. Systems Theory 6 (1972), 164-192.

[5] B. de Mathan, Irrationality measures and transcendence in positive characteristic, J. Number Theory 54 (1995), 93-112. 
[6] M. Koskas, Complexité de suites et fonction de Carlitz, thèse, Université de Bordeaux I, 1995.

[7] L. Kuipers and H. Niederreiter, Uniform Distribution of Sequences, Wiley, 1974.

[8] W. H. Mills and D. P. Robbins, Continued fractions for certain algebraic power series, J. Number Theory 23 (1986), 388-404.

[9] M. Mkaouar, Sur le développement en fraction continue de la série de Baum et Sweet, Bull. Soc. Math. France 123 (1995), 361-374.

Département de Mathématiques

Université Bordeaux I

351, Cours de la Libération

33405 Talence Cedex, France

E-mail: yao@math.u-bordeaux.fr 